\title{
Lithium abundances in metal-poor stars ${ }^{\star}$ (Research Note)
}

\author{
J. R. Shi ${ }^{1,2}$, T. Gehren ${ }^{2}$, H. W. Zhang ${ }^{2,3}$, J. L. Zeng ${ }^{1,2}$, and G. Zhao ${ }^{1}$ \\ ${ }^{1}$ National Astronomical Observatories, Chinese Academy of Sciences, Beijing 100012, PR China \\ e-mail: sjr@bao.ac.cn \\ 2 Institut für Astronomie und Astrophysik der Universität München, Scheinerstrasse 1, 81679 München, Germany \\ ${ }^{3}$ Peking University, Beijing 100871, PR China
}

Received 7 November 2006 / Accepted 2 January 2007

\section{ABSTRACT}

\begin{abstract}
Aims. Lithium abundances for 19 metal-poor stars are determined using high-resolution spectroscopy. The abundances of stars on the lithium plateau are discussed.

Methods. All abundance results are derived from NLTE statistical equilibrium calculations and spectrum synthesis methods. Results. In agreement with previous analyses it is found that excitation and de-excitation due to hydrogen collisions are negligible for the lithium line formation process, while charge transfer reactions are an important source of thermalization. However, the resulting NLTE effects on the determination of lithium abundances for metal-poor stars are negligible $(<0.06 \mathrm{dex})$.

Conclusions. The mean lithium abundance for stars on the lithium plateau determined from NLTE analyses is $A(\mathrm{Li}) \sim 2.26$, while it is 2.21 dex when charge transfer reactions are included. The latter result enhances the discrepancy between the observed lithium abundances and the primordial lithium abundance as inferred by the WMAP analysis of the cosmic microwave background. This discrepancy may be explained by metal diffusion.
\end{abstract}

Key words. line: formation - line: profile - stars: abundance - stars: late-type - galaxies: evolution

\section{Introduction}

Lithium abundances in metal-poor stars are of importance for cosmology, the evolution of the Galaxy, Big Bang nucleosynthesis and stellar structure. Spite \& Spite (1982) first detected that the ${ }^{7} \mathrm{Li}$ abundance in halo dwarfs is independent of temperature and metallicity, suggesting that they represent the primordial abundance from Big Bang nucleosynthesis (BBN). However, based on the WMAP results (Wilkinson Microwave Anisotropy Probe, see Spergel et al. 2003), the BBN prediction for primordial ${ }^{7} \mathrm{Li}$ is between 2 and 3 times higher than observed in the atmospheres of halo dwarfs (Coc et al. 2004; Cuoco et al. 2004; Cyburt 2004; Cyburt et al. 2005), and it remains to be resolved what type of systematic effects are responsible for this discrepancy.

Norris et al. (1994) and Thorburn (1994) have found a dependence of $A(\mathrm{Li})$ on both temperature and metallicity, which was confirmed by later work, such as that of Ryan et al. (1996, 1999, 2001), Zhang \& Zhao (2003), and Boesgaard et al. (2005). However, Molaro et al. (1995) and Bonifacio \& Molaro (1997) questioned this trend. Their result is also supported by Meléndez \& Ramírez (2004) and Charbonnel \& Primas (2005). The latter authors noted that for some stars, common to several analyses, very discrepant $\mathrm{Li}$ abundances were reported, which could have influenced some of the early claims for a significant abundance dispersion. They suggested that this could also play a role in the current debate about the existence of a slope of lithium abundances with temperature and metallicity.

* Based on observations collected at the Germany-Spanish Astronomical Center, Calar Alto, Spain.
As Li I has a low ionization potential, non-local thermodynamic equilibrium (NLTE) effects can be expected to be important (Carlsson et al. 1994; Asplund et al. 2003). Carlsson et al. (1994) have shown that the LTE approximation - when deriving lithium abundances for cool stars - is not representative of the physical properties of the atmospheric layers in which the $6707 \AA$ line forms. Since NLTE corrections vary in sign and size when spanning a large range of stellar parameters, ignoring these corrections may affect any interpretation of the $A(\mathrm{Li})$-plateau and its possible slope with effective temperature and metallicity. Recently, Barklem et al. (2003) found that the collisional excitation and de-excitation $\left(\mathrm{Li}^{*}+\mathrm{H} \rightleftharpoons \mathrm{Li}^{*}+\mathrm{H}\right)$ reactions are negligible for the line formation process, while the charge transfer reactions $\left(\mathrm{Li}^{*}+\mathrm{H} \rightleftharpoons \mathrm{Li}^{+}+\mathrm{H}^{-}\right)$are important in thermalizing lithium. Asplund (2005) suggested that new 1D calculation including the charge transfer data and improved line-blocking treatment would be worthwhile.

In this work we present new results of NLTE studies for metal-poor stars. In Sect. 2 we comment on the observational techniques. The models are discussed in Sect. 3, while in Sect. 4 we describe the methods of analysis and uncertainty for our results. The results and discussions are given in Sect. 5, and the summary is presented in the last section.

\section{Observations}

The spectra of our samples were obtained from 1997 to 2003 with the fiber-coupled Cassegrain échelle spectrograph FOCES (Pfeiffer et al. 1998) mounted on the $2.2 \mathrm{~m}$ telescope of the Calar Alto Observatory. The standard configuration was a $2048^{2}$ $24 \mu$ CCD that covered the wavelength region 3900-9000 


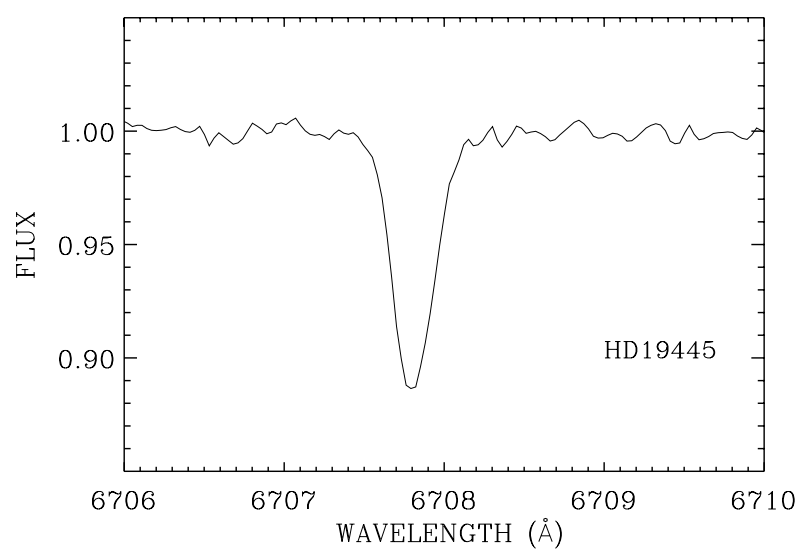

Fig. 1. The Li I line near $\lambda 6707 \AA$ in the program star HD 19445.

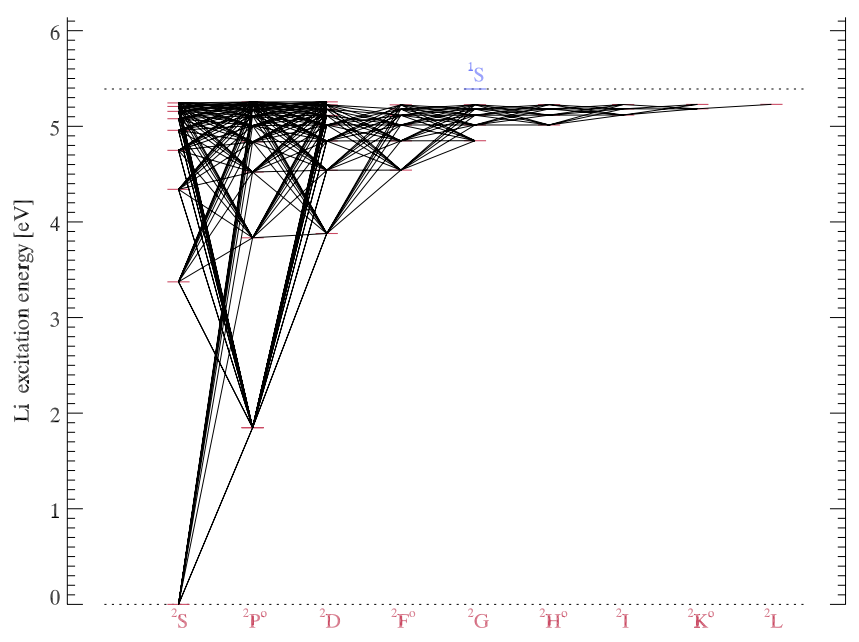

Fig. 2. Grotrian diagram for Li I.

with $\lambda / \Delta \lambda \sim 40000$. Some stars were observed with the $15 \mu$ CCD resulting in $R \sim 65000$. All stars were observed at least twice with a signal-to-noise ratio ranging from $S / N \sim 100$ up to $S / N \sim 400$. Our sample contains main sequence/turnoff stars presented in Gehren et al. (2004, 2006).

Data extraction followed the standard automatic IDL program environment designed for the FOCES spectrograph (Pfeiffer et al. 1998). All echelle images including flatfield and ThAr were corrected for bias and scattered light background. Objects and ThAr exposures were extracted and corrected for flatfield response. Bad pixels were detected and as far as possible removed by comparison of at least 2 single exposures (see Gehren et al. 2004, for details). Figure 1 shows a portion of the spectrum of HD 19445 around $6707 \AA$.

\section{NLTE line formation}

\subsection{Model atom}

The lithium model atom employed in this paper is illustrated by its Grotrian diagram in Fig. 2. We extend the number of levels to $n=10$. The model is closed with the $\mathrm{Li}^{+}$ground state containing 49 levels and all transitions between them. Fine structure splitting is neglected with the exception of $2 \mathrm{p}^{2} \mathrm{P} 1 / 2^{\circ}$ and $2 \mathrm{p}^{2} \mathrm{P} 3 / 2^{\circ}$, and a balance between these two levels is assumed.

The level energies and oscillator strengths are taken from the TOPBASE data when available. All other energy levels and transition probabilities are assumed to follow a hydrogen-like approximation. We adopt the photoionization cross-sections provided by the Opacity Project via the TOPBASE system (Cunto $\&$ Mendoza 1992) for the 25 terms available; for the remaining terms hydrogen-like approximations are used. As usual, background opacities are calculated with an opacity sampling code based on the line lists made available by Kurucz (1992).

As in previous calculations, all bound-bound electron collision strengths for allowed transitions are approximated by the simple formula of van Regemorter (1962). Forbidden collisions in our representation follow Allen's (1973) formula for which no experimental verification is available. Ionization by electronic collisions is calculated from Seaton's (1962) classical path approximation. The collision rate is therefore proportional to the photoionization cross-section at the threshold.

The cross-sections for excitation and de-excitation due to collisions with neutral hydrogen are taken from Drawin (1968, 1969); here we adopt a multiplication factor of $S H=0.05$. As already noted by Asplund et al. (2003) and Barklem et al. (2003), hydrogen collisions are negligible for the $\mathrm{Li}$ line formation process. The cross-sections for charge transfer reactions are taken from Barklem et al. (2003).

\subsection{Model atmospheres}

Our analyses are all based on the ODF line-blanketed LTE model atmospheres generated and discussed by Fuhrmann et al. (1997). Their main characteristics are:

- the iron opacity was calculated with the improved meteoritic value $\log \varepsilon \mathrm{Fe}=7.51$ (Anders \& Grevesse 1989);

- opacities due to $\alpha$-elements (O, Ne, Mg, Si, S, Ar, Ca and Ti) for metal-poor stars $([\mathrm{Fe} / \mathrm{H}]<-0.6)$ were calculated with the $\alpha$-element abundances enhanced by 0.4 dex;

- the mixing-length parameter $l / H p$ was adopted to be 0.5 , in order to determine consistent temperatures for $\mathrm{H} \alpha$ and the higher Balmer lines.

\subsection{Stellar parameters}

For most of the stars we adopt the stellar parameters determined by Korn et al. (2003) and Gehren et al. (2006), where the effective temperatures are derived from the wings of the Balmer lines; surface gravities are based on the HIPPARCOS parallaxes. Iron abundances are based on Fe II lines, and the microturbulence velocities are estimated by requiring that the iron abundances derived from Fe II lines should not depend on equivalent width. The uncertainties for the temperature, surface gravity, metal abundance and microturbulence velocities are generally assumed to be $\pm 80 \mathrm{~K}, 0.05 \mathrm{dex}, 0.05 \mathrm{dex}$ and $0.1 \mathrm{~km} \mathrm{~s}^{-1}$ respectively.

Following previous work, we employ the macroturbulence parameters $\zeta$ in the radial-tangential form and adopt values as described in Gray (1984). The projected rotational velocity is obtained as the residual to the observed line profiles, after having removed the known instrumental profile obtained from the Moon spectra. It is, however, not used in this analysis.

\subsection{Atomic line data}

We adopt the oscillator strengths from the NIST data base, namely $\log g f=0.002$ and -0.299 for the 6707.76 and $6707.91 \AA$ lines, respectively. While collisional broadening parameters describing van der Waals interaction with hydrogen atoms are taken from Barklem et al. (1998), we note that the lines are not sensitive to the damping constant either. 
Table 1. Atmospheric parameters and lithium abundances.

\begin{tabular}{|c|c|c|c|c|c|c|c|}
\hline Star & $\begin{array}{c}T_{\text {eff }} \\
\mathrm{K}\end{array}$ & $\log g$ & {$[\mathrm{Fe} / \mathrm{H}]$} & $\begin{array}{c}\xi \\
\mathrm{km} \mathrm{s}^{-1}\end{array}$ & $\begin{array}{r}A(\mathrm{Li}) \\
\mathrm{LTE}\end{array}$ & $\begin{array}{l}A(\mathrm{Li}) \\
\text { NLTE }\end{array}$ & $\begin{array}{c}A(\mathrm{Li}) \\
\mathrm{NLTE}(\mathrm{CH})\end{array}$ \\
\hline BD-04 3208 & 6310 & 3.98 & -2.23 & 1.50 & 2.32 & 2.31 & 2.26 \\
\hline $\mathrm{BD}+541216$ & 6015 & 4.29 & -1.57 & 1.40 & 2.25 & 2.24 & 2.19 \\
\hline G $20-8$ & 6115 & 4.20 & -2.19 & 1.50 & 2.30 & 2.30 & 2.23 \\
\hline G $165-39$ & 6220 & 3.96 & -2.00 & 1.90 & 2.24 & 2.22 & 2.18 \\
\hline G $212-7$ & 5560 & 4.77 & -1.60 & 1.30 & 1.60 & 1.60 & 1.56 \\
\hline G $119-32$ & 5715 & 4.39 & -1.88 & 1.20 & 2.12 & 2.14 & 2.09 \\
\hline G $84-29$ & 6240 & 4.14 & -2.45 & 1.60 & 2.19 & 2.19 & 2.15 \\
\hline G $188-22$ & 6040 & 4.37 & -1.25 & 1.50 & 2.26 & 2.24 & 2.21 \\
\hline G $242-4$ & 5815 & 4.31 & -1.10 & 1.20 & 1.98 & 2.00 & 1.95 \\
\hline HD 19445 & 6032 & 4.40 & -2.08 & 1.50 & 2.28 & 2.27 & 2.21 \\
\hline HD 45282 & 5282 & 3.12 & -1.52 & 1.40 & 1.18 & 1.29 & 1.19 \\
\hline HD 84937 & 6346 & 4.00 & -2.16 & 1.83 & 2.28 & 2.27 & 2.21 \\
\hline HD 94028 & 5925 & 4.19 & -1.51 & 1.50 & 2.21 & 2.21 & 2.15 \\
\hline HD 140283 & 5806 & 3.69 & -2.42 & 1.50 & 2.23 & 2.28 & 2.18 \\
\hline HD 188510 & 5490 & 4.51 & -1.67 & 1.21 & 1.61 & 1.63 & 1.58 \\
\hline HD 194598 & 6040 & 4.30 & -1.16 & 1.34 & 2.27 & 2.27 & 2.22 \\
\hline HD 201891 & 5943 & 4.24 & -1.05 & 1.18 & 2.21 & 2.21 & 2.16 \\
\hline HD 284248 & 6202 & 4.23 & -1.61 & 1.55 & 2.34 & 2.33 & 2.29 \\
\hline HD 338529 & 6280 & 3.93 & -2.25 & 2.00 & 2.23 & 2.24 & 2.19 \\
\hline
\end{tabular}

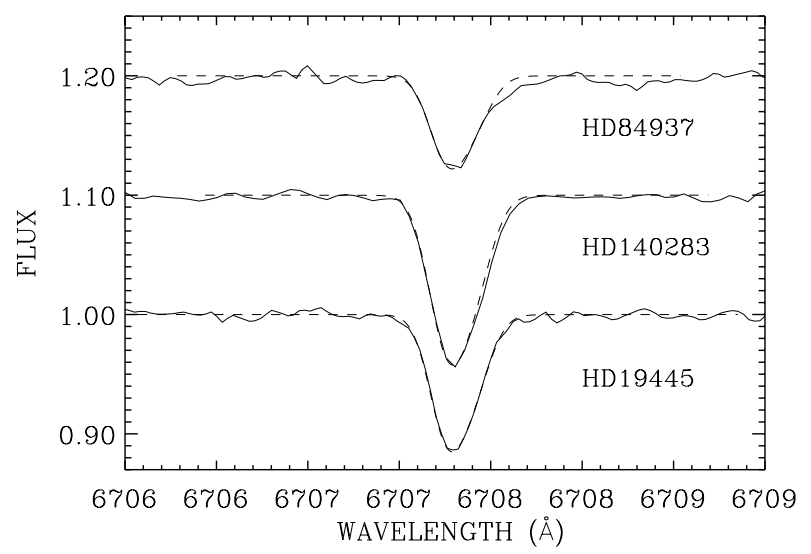

Fig. 3. Synthetic NLTE flux profiles of Li lines compared with the observed spectra. The solid lines are the observed spectra, while the dashed lines are NLTE profiles.

\section{Lithium abundances}

\subsection{Abundance determination}

Abundance determinations are carried out using a spectral synthesis method based on the IDL/Fortran SIU software package of Reetz (1993). The synthetic spectra are convolved with macroturbulence, rotational and instrumental broadening profiles in order to match the observed spectral lines. Lithium abundances are obtained with a best fit to the observed line profile. They are listed in Table 1, together with the stellar parameters. Three different sets of lithium abundances are reported for each star, namely LTE and NLTE, the latter without and with charge reaction processes. The internal errors in the derived lithium abandances are less than 0.05 dex. An example of comparison of synthetic profiles with the observed ones for lithium lines is shown in Fig. 3.

Without the charge reactions, our results show that the NLTE corrections are negligible (about $0.02 \mathrm{dex}$ ) for stars on the

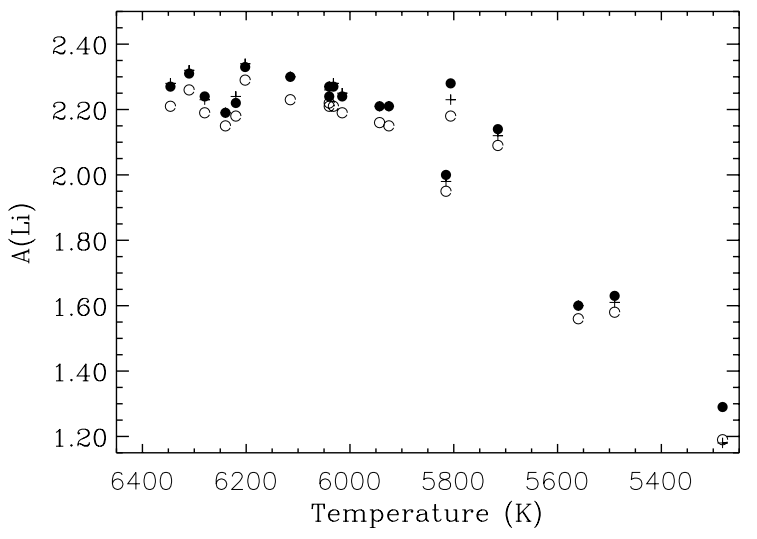

Fig. 4. Li abundances as a function of temperature. Pluses (+) are the LTE lithium abundances; filled circles $(\bullet)$ are the NLTE lithium abundances without charge transfer and open circles ( $)$ ) are the lithium abundances with charge transfer.

plateau, while they are about 0.1 dex for low temperature metalpoor main sequence and subgiant stars, i.e., HD 45282, and they change their sign with the stellar parameters. Due to the overpopulation of the ground state, the NLTE effects are all negative, and they decrease the lithium abundances of the plateau stars by about -0.05 dex when charge reactions are included.

\subsection{Uncertainty in fundamental parameters}

It is known that lithium abundances are very sensitive to the effective temperature chosen, whereas their dependence on the other parameters, i.e. gravity, metallicity and microturbulence is negligible. Common uncertainties on $\log g,[\mathrm{Fe} / \mathrm{H}]$ and $\xi$ $\left( \pm 0.07 \mathrm{dex}, \pm 0.05 \mathrm{dex}\right.$ and $\pm 0.1 \mathrm{~km} \mathrm{~s}^{-1}$, respectively) will not affect the final lithium abundances. Fields et al. (2005) discussed the influence of the temperature scale for halo dwarfs on lithium abundances and chemical evolution in detail. An uncertainty of $\pm 80 \mathrm{~K}$ in $T_{\text {eff }}$ for stars of our sample translates into about \pm 0.06 dex in the lithium abundance.

\section{Results and discussion}

Lithium abundances of metal-poor stars have been determined by several groups. We have 16 stars in common with Charbonnel \& Primas (2005) and five stars with Meléndez \& Ramírez (2004). The average differences between our $[\mathrm{Li} / \mathrm{H}]$ and theirs are $0.10 \pm 0.10$ and $-0.08 \pm 0.07$ for NLTE without the charge reactions. We note that the temperatures adopted for our sample stars are slightly higher than those of Charbonnel \& Primas $(+33 \pm 110 \mathrm{~K})$, but lower than determined by Meléndez \& Ramírez $(-51 \pm 112.52 \mathrm{~K})$; part of the differences can be explained by the different temperatures adopted. We have four stars in common with Asplund et al. (2006) and two stars with Boesgaard et al. (2005); the average differences between our $[\mathrm{Li} / \mathrm{H}]$ and theirs are $0.07 \pm 0.04$ and $0.03 \pm 0.03$, respectively. Though not perfect, the agreement is satisfactory.

Figure 4 shows the overall distribution of $A(\mathrm{Li})$ in our sample as a function of temperature. It is obvious that the lithium abundances depend on temperatures for stars with $T_{\text {eff }}<6000 \mathrm{~K}$, whereas no such trend can be found for stars with $T_{\text {eff }}>6000 \mathrm{~K}$.

The distribution of $A(\mathrm{Li})$ with $[\mathrm{Fe} / \mathrm{H}]$ for all stars in the sample is shown in Fig. 5, while Fig. 6 is restricted only to stars with $T_{\text {eff }}$ greater than $6000 \mathrm{~K}$. From these two figures, it is clearly 


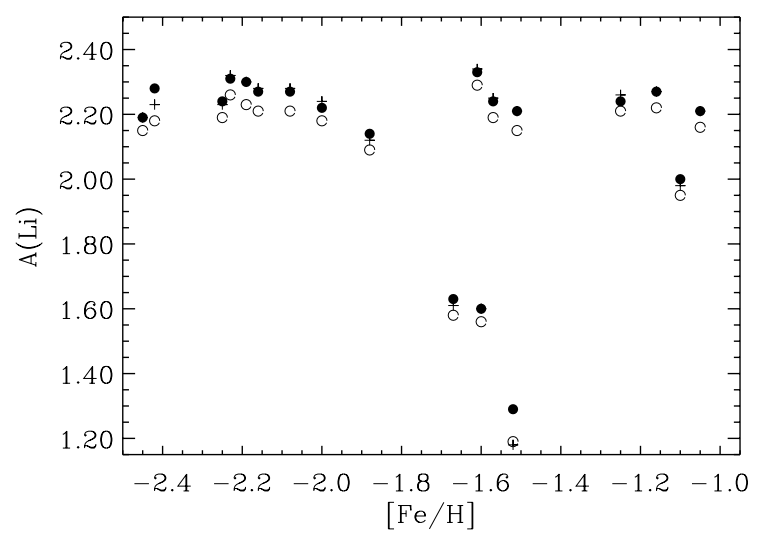

Fig. 5. $A(\mathrm{Li})$ abundances analysis as a function of $[\mathrm{Fe} / \mathrm{H}]$. Symbols are the same as in Fig. 4.

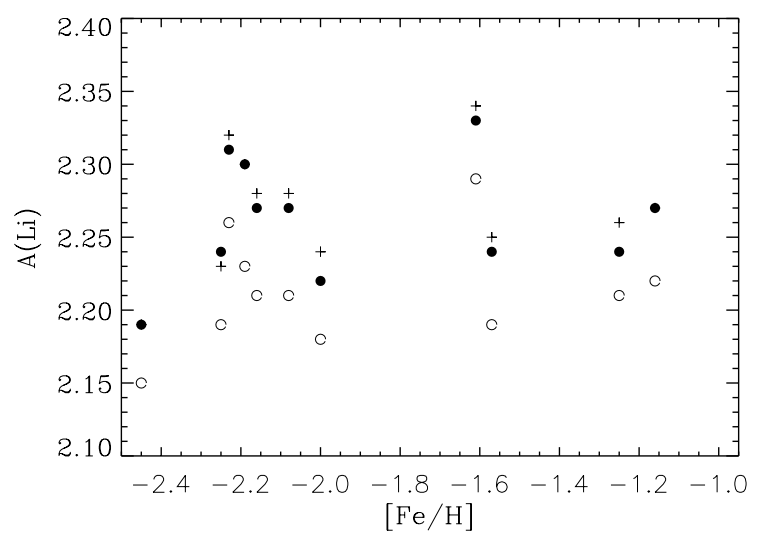

Fig. 6. Same as Fig. 3, but only with the stars with $T_{\text {eff }}>6000 \mathrm{~K}$. Symbols are the same as in Fig. 4.

seen that $A(\mathrm{Li})$ is independent of metallicity for our sample. The abundances obtained in this work for plateau stars show a very small star-to-star scatter. This is a consequence of employing internally very accurate temperatures based on the Balmer lines. For stars with $T_{\text {eff }}>6000 \mathrm{~K}$, the mean $A(\mathrm{Li})$ is $2.27 \pm 0.04$, $2.26 \pm 0.04$ and $2.21 \pm 0.04$ for LTE, and for NLTE without and with charge reactions, respectively. Our Li abundance is higher than that of Ryan et al. (1999), but lower than that of Meléndez \& Ramírez (2004).

When charge reactions are included, our NLTE results are in good agreement with those of Barklem et al. (2003), namely the derived lithium abundances are lowered by 0.05 ...0.10 dex. This trend enhances the discrepancy between the observed lithium abundances in metal-poor stars and the primordial lithium abundance as inferred from the WMAP analysis of the cosmic microwave background. As often discussed, a possible reason may be the depletion of atmospheric ${ }^{7} \mathrm{Li}$. Due to turbulent mixing, mass loss and/or atomic diffusion, uniform depletion factors of order 0.2 dex (Vauclair \& Charbonnel 1998) and as high as 0.3 dex (Salaris \& Weiss 2001; Richard et al. 2005) have been discussed. This possibility faces the strong constraint that the observed lithium abundances show very little dispersion. It is unlikely that the stellar processes that depend on temperature, mass, and rotation velocity of the star would destroy ${ }^{7} \mathrm{Li}$ always by the same amount. Coc et al. (2004) suggested that the ${ }^{7} \mathrm{Be}(\mathrm{d}, \mathrm{p}) 2 \alpha$ reaction would solve the lithium problem if its crosssection were very much higher than assumed. However, recent experimental results show this cross-section is ten times overestimated (Angulo et al. 2005).
Recently, Korn et al. (2006) suggested that metal diffusion may be responsible for the low apparent stellar lithium abundances in the atmospheres of old stars. Their atomic diffusion model, added to standard stellar evolution models, leads to a decrease of the original lithium abundance by about 0.25 dex. At the time of formation of these very old stars, therefore, the cosmic matter would have had a lithium abundance of $A(\mathrm{Li}) \simeq 2.21+0.25=2.46 \pm 0.04$, a value in good agreement with the primordial lithium abundance based on the cosmic microwave background and Big Bang nucleosynthesis, A(Li) $\mathrm{WMAP} \simeq 2.54 \pm 0.10$.

\section{Conclusions}

We have determined the lithium abundances of 19 metal-poor stars spanning the range $-2.5<[\mathrm{Fe} / \mathrm{H}]<-1.0$. All abundances are derived from NLTE statistical equilibrium calculations. Based on our results, we come to the following conclusions.

1. For plateau stars our lithium abundances are independent of the temperatures and metallicities.

2. The lithium abundances obtained in this work for plateau stars show a very small star-to-star scatter. The mean lithium abundance obtained for the plateau stars are $A(\mathrm{Li})=2.26$ and 2.21 dex without and with the charge reactions included. Our result is higher than the primordial lithium abundance proposed by Ryan et al. (2001) $(A(\mathrm{Li}) \approx 2.0 \ldots 2.1 \mathrm{dex})$, while it is lower than that obtained by Meléndez \& Ramírez (2004).

3. NLTE corrections are relatively small $(<0.1$ dex $)$. NLTE abundances are decreased compared to LTE by about -0.05 for all sample stars when charge reactions are included.

4. While metal diffusion seems to remove most of the problems related to the cosmic lithium abundance plateau, it is important to note the remaining influence of 3D hydrodynamic models on the final value of the lithium abundance.

Acknowledgements. S.J.R. thanks the Institute of Astronomy and Astrophysics of Munich University for warm hospitality during a productive stay in 2004 . We thank the referee for helpful comments. This research was supported by the National Natural Science Foundation of China under grants Nos. 10433010 and 10521001, and by the Deutsche Forschungsgemeinschaft under contracts Ge 490/26-1 and 446 CHV112.

\section{References}

Allen, C. W. 1973, Astrophysical quantities, 3rd edn. (London: Athlone Press) Anders, E., \& Grevesse, N. 1989, Geochim. Cosmochim. Acta, 53, 197 Angulo, C., Casarejos, E., Couder, M., et al. ApJ, 630, L105

Asplund, M. 2005, ARA\&A, 43, 481

Asplund, M., Carlsson, M., \& Botnen, A. V. 2003, A\&A, 399, L31 Asplund, M., Lambert, D. L., Nissen, P. E., Primas, F., \& Smith, V. V. 2006, ApJ, 644,229

Barklem, P. S., O’Mara, B. J., \& Ross, J. E. 1998, MNRAS, 296, 1057

Barklem, P. S., Belyaev, A. K., \& Asplund, M. 2003, A\&A, 409, L1

Boesgaard, A. M., Stephens, A., \& Deliyannis, C. P. 2005, ApJ, 633, 398

Bonifacio, P., \& Molaro, P. 1997, MNRAS, 285, 847

Carlsson, M., Rutten, R. J., Bruls, J. H. M. J., \& Shchukina, N. G. 1994, A\&A, 288,860

Charbonnel, C., \& Primas, F. 2005, A\&A, 442, 961

Coc, A., Vangioni-Flam, E., Descouvemont, P., Adahchour, A., \& Angulo, C. 2004, ApJ, 600, 544 
Cunto, W., \& Mendoza, C. 1992, Rev. Mex. Astrofis, 23, 107

Cuoco, A., Iocco, F., Mangano, G., et al. 2004, IJMPA, 19, 4431

Cyburt, R. H. 2004, Phys. Rev. D, 70, 3505

Cyburt, R. H., Fields, B. D., Olive, K. A., Skillman, E. 2005, Astropart. Phys., 23,313

Drawin, H. W. 1968, Z. Phys., 211, 404

Drawin, H. W. 1969, Z. Phys., 225, 483

ESA 1997, The Hipparcos and Tycho Catalogues, ESA, SP-1200

Fields, B. D., Olive, K. A., \& Vangioni-Flam, E. 2005, ApJ, 623, 1083

Fuhrmann, K., Pfeiffer, M., Frank, C., Reetz, J., \& Gehren, T. 1997, A\&A, 323, 909

Gehren, T., Butler, K., Mashonkina, L., Reetz, J., \& Shi, J. R. 2001, A\&A, 366, 981

Gehren, T., Liang, Y. C., Shi, J. R., Zhang, H. W., \& Zhao, G. 2004, A\&A, 413, 1063

Gehren, T., Shi, J. R., Zhang, H. W., Zhao, G., \& Korn, A. J. 2006, A\&A, 451, 1065

Gray, D. F. 1984, ApJ, 281, 719

Korn, A. J., Shi, J. R., \& Gehren, T. 2003, A\&A, 407, 691

Korn, A. J., Grundahl, F., Richard, O., et al. 2006, Nature, 442, 657

Kurucz, R. L. 1992, Rev. Mex. Astron. Astrofis. 23, 45
Meléndez, J., \& Ramírez, I. 2004, ApJ, 615, L33

Molaro, P., Primas, F., \& Bonifacio, P. 1995, A\&A, 295, L47

Molaro, P., Bonifacio, P., Castelli, F., \& Pasquini, L. 1997, A\&A, 319, 593

Norris, J. E., Ryan, S. G., \& Stringfellow, G. S. 1994, ApJ, 423, 386

Pfeiffer, M. J., Frank, C., Baumüller, D., Fuhrmann, K., \& Gehren, T. 1998 A\&AS, 130, 381

Reetz, J. K. 1993, unpublished

Richard, O., Michaud, G., \& Richer, J. 2005, ApJ, 619, 538

Ryan, S. G., \& Deliyannis, C. P. 1998, ApJ, 500, 398

Ryan, S. G., Beers, T. C., Deliyannis, C. P., \& Thorburn, J. A. 1996, ApJ, 458, 543

Ryan, S. G., Norris, J. E., \& Beers, T. C. 1999, ApJ, 523, 624

Ryan, S. G., Kajino, T., Beers, T. C., et al. 2001, ApJ, 549, 55

Salaris, M., \& Weiss, A. 2001, A\&A, 376, 955

Seaton, M. J. 1962, in Atomic and Molecular Processes (New York: Acad. Press)

Spergel, D. M., Verde, L., \& Peiris, H. V. 2003, ApJS, 148, 175

Spite, F., \& Spite, M. 1982, A\&A, 115, 357

Thorburn, J. A. 1994, ApJ, 421, 318

Vauclair, S., \& Charbonnel, C. 1998, ApJ, 502, 372

van Regemorter, H. 1962, ApJ, 136, 906

Zhang, H. W., \& Zhao, G. 2003, ChJAA, 3, 453 